\title{
Evaluation of Mode Meters Robust to Forced Oscillations using Field-Measured Data
}

\author{
Jim Follum, Urmila Agrawal, and Pavel Etingov \\ Pacific Northwest National Laboratory \\ james.follum@pnnl.gov
}

\begin{abstract}
Mode meters are used by power system operators to monitor a system's small-signal stability by estimating the system's electromechanical modes of oscillation. When a system undergoes a forced oscillation, mode meters may become biased because the two types of oscillation cannot be distinguished. Modified mode meter algorithms robust to this bias were proposed in prior research, but these studies were based primarily on simulated data. In this paper, modified least squares and Yule-Walker mode meter algorithms are evaluated using field-measured data from phasor measurement units (PMUs). Results show that the sensitivities of the least squares algorithm make it impractical for use given the complexities of real-world forced oscillations. However, the modified Yule-Walker algorithm is shown to perform well and has significant potential for practical deployment in mode meters.
\end{abstract}

\section{Introduction}

Reliable power system operation requires that small-signal stability be maintained. Systems become unstable when one or more inter-area electromechanical modes of oscillation become negatively damped. The modes govern oscillatory exchanges of energy between generators in different parts of the system, so when a mode is negatively damped it results in growing oscillations in frequency and power flow. The oscillations will continue to grow until the system is returned to stability or system breakup occurs. The August 10, 1996 breakup of the western North American power system (WNAPS) is a well-known example of a system losing small-signal stability [1].

Following the 1996 event, tools called mode meters were developed to continuously track the frequency and

The Pacific Northwest National Laboratory is operated for the U.S. Department of Energy by Battelle Memorial Institute under Contract DE-AC05-76RL01830. This work was funded by the U.S. DOE. damping of inter-area electromechanical modes. These tools rely on the synchrophasor measurements from phasor measurement units (PMUs), which capture the dynamic response of the system to random load changes [2]. Mode meters are now commercially available and in use by system operators $[3,4,5]$.

Along with natural oscillations related to inter-area electromechanical modes, forced oscillations (FOs) are common in power systems. While natural oscillations are related to the system's dynamics, FOs arise when a piece of equipment injects an oscillation into the system. The resulting oscillations typically exhibit a sustained amplitude as long as the forcing input is present. Several examples can be found in [4], including oscillations due to a wind power plant's generator controller, a hydro unit running in its rough zone, and erroneous measurements feeding a generator's controller.

Forced oscillations can bias mode meters when their frequency is close to an inter-area electromechanical mode of oscillation. Bias occurs when the meter cannot distinguish the sustained FO from a poorly damped natural oscillation. See [5] for a real-world example of a mode meter's damping estimate dropping due to the presence of a FO. It is important to note that the FO did not actually cause the system's damping to decline in this example. The actual damping of the natural mode of oscillation remained relatively steady, while the estimate of the damping ratio became severely biased.

Solutions to this problem are proposed in $[6,7,8]$. The methods in [8] examine input measurements for any oscillation and do not distinguish between FOs and modal oscillations. In contrast, the authors of [6] and [7] modify the AutoRegressive Moving Average (ARMA) model to explicitly represent FO components of the input measurements. The system's electromechanical modes and FOs are distinguished, but they are estimated simultaneously by applying modified least squares (LS) [6] and Yule-Walker (YW) [7] algorithms. Following the convention in $[6,7]$, the modified algorithms will be denoted as $\mathrm{LS}+\mathrm{S}$ and $\mathrm{YW}+\mathrm{S}$. The $+\mathrm{S}$ denotes the addition of a sinusoid term to the ARMA model. 
While the methods in $[6,7,8]$ each appear to hold promise, they have so far been evaluated primarily with simulated PMU data. In this paper, the methods of [6] and [7] are evaluated using field-measured PMU data from four different FO events. Though the underlying LS and YW methods are closely related and performed similarly in prior simulation studies, the results reveal important differences in sensitivity to real-world conditions. These results demonstrate the practical importance of addressing the problem and have implications for making the tools commercially available. Another key contribution of this paper is documenting many of the details that led to successful implementation. In addition, a new method of estimating the timing of a FO's start and end, which is necessary for the $\mathrm{LS}+\mathrm{S}$ method, is introduced. The new method is more practical in real-world conditions than the algorithm proposed in [9].

The rest of the paper is organized as follows. In Section 2, an overview of the $\mathrm{LS}+\mathrm{S}$ and $\mathrm{YW}+\mathrm{S}$ algorithms of [6] and [7] are provided. Considerations for real-world implementations of the methods are discussed in Section 3. Results from the four field-measured FO event cases are provided in Section 4 , followed by a discussion of conclusions in Section 5 .

\section{Overview of Mode Meter Algorithms}

In this section, overviews of the algorithms in [6] and [7] are provided. These algorithms were designed to provide reliable mode estimates even in the presence of FOs. They do this by incorporating FOs into the black-box model representing the system's dynamics. The resulting ARMA plus sinusoid (ARMA+S) model is depicted in Fig. 1. Here $e(k)$ represents aggregate random load changes perturbing the system's dynamics at discrete time $k$, and $y(k)$ denotes the system's measured response. The FO input is modeled as

$$
s(k)=\sum_{p=1}^{P} A_{p} \cos \left(\omega_{p} k+\delta_{p}\right) I_{\left[\epsilon_{p}, \eta_{p}\right]}(k)
$$

where $A_{p}, \omega_{p}$, and $\delta_{p}$ are, respectively, the amplitude, frequency in radians per sample, and phase in radians of the $p^{t h}$ sinusoid in the sum. The starting sample, $\epsilon_{p}$, and ending sample, $\eta_{p}$, of the $p^{\text {th }}$ sinusoid are included via the indicator function

$$
I_{[\epsilon, \eta]}(k)= \begin{cases}1, & 0 \leq \epsilon \leq k \leq \eta \leq K-1 \\ 0, & \text { else }\end{cases}
$$

To simplify notation, the indicator function corresponding to the $p^{t h}$ sinusoid will be abbreviated

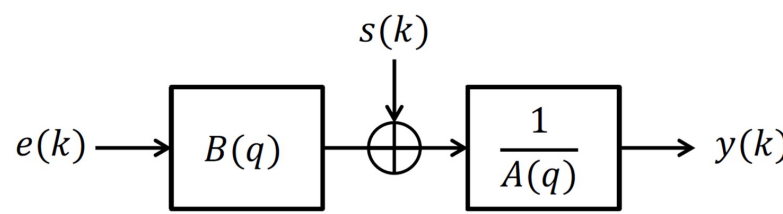

Figure 1. Block diagram of the ARMA+S model structure

as $I_{p}(k)$ when the starting and ending samples are not of immediate interest. Note that (1) can be used to represent any periodic signal.

The autoregressive (AR) and moving average (MA) portions of the model are represented by the $A(q)$ and $B(q)$ polynomials, respectively, where $q$ is the shift operator such that $q^{-\tau} y(k)=y(k-\tau)$. The system's electromechanical modes correspond to the discrete-time poles of the ARMA+S system:

$$
z_{i}=\operatorname{roots}(A(q)) .
$$

The continuous-time poles follow as

$$
\lambda_{i}=\ln \left(z_{i}\right) \times f_{s}
$$

where $f_{s}$ is the sampling rate of the data. Letting $\lambda_{i}=$ $\sigma_{i}+j \omega_{i}$, the frequency in units of $\mathrm{Hz}$ and damping ratio of the mode can be expressed as

$$
f_{i}=\frac{\omega_{i}}{2 \pi} H z
$$

and

$$
\zeta_{i}=-\frac{\sigma_{i}}{\sqrt{\sigma_{i}^{2}+\omega_{i}^{2}}} \times 100 \% .
$$

The $\mathrm{LS}+\mathrm{S}$ and $\mathrm{YW}+\mathrm{S}$ algorithms allow the $\mathrm{AR}$ polynomial to be estimated. Equations (3)-(6) are then used to estimate the frequencies and damping ratios of dominant inter-area electromechanical modes. Overviews of the modified algorithms are provided in the following subsections.

\subsection{Modified Least Squares Algorithm}

Here, an overview of the detailed derivation in [6] is provided. To begin, note that the measured output of the ARMA+S model can be expressed as the constant coefficient difference equation

$$
y(k)=-\sum_{i=1}^{n_{a}} a_{i} y(k-i)+\sum_{i=0}^{n_{b}} b_{i} e(k-i)+s(k)
$$

where the AR and MA coefficients are denoted as $a_{i}$ and $b_{i}$, respectively. Writing this equation out for several 
values of $k$ and placing in matrix notation leads to

$$
\underline{y}=\left[\begin{array}{ccc}
Y & E & S \circ I
\end{array}\right] \underline{\theta}+\underline{e}
$$

where $y$ and $Y$ are, respectively, a vector and matrix containing measurements, $E$ is a matrix containing estimates of $e(k), S$ is a matrix of $\sin$ and cos terms with frequencies of the $\mathrm{FO}$, $\circ$ indicates the Hadamard product (element-by-element multiplication), $I$ contains indicator functions specifying the start and end samples of the FO, $\underline{\theta}$ is a parameter vector containing the AR polynomial coefficients, and $\underline{e}$ is a vector containing $e(k)$. The least squares solution to $(8)$ is

$$
\underline{\hat{\theta}}=\left[\begin{array}{ccc}
Y & E & S \circ I
\end{array}\right]^{\dagger} \underline{y}
$$

where $\dagger$ denotes the pseudoinverse. The frequencies and damping ratios of dominant inter-area electromechanical modes are estimated by applying (3)-(6) with the AR polynomial estimates contained in $\underline{\hat{\theta}}$.

The solution in (9) requires estimates of the frequency, start time, and end time of each FO. Methods for estimating these parameters are discussed in $[9,10]$. Further details regarding practical implementation are discussed in Section 3.

\subsection{Modified Yule-Walker Algorithm}

An overview of the $\mathrm{YW}+\mathrm{S}$ algorithm proposed in [7] is provided here. To begin, consider the autocovariance sequence of the measured data given by

$$
r(k, \gamma)=E\{y(k) y(k-\gamma)\}
$$

where $E\{\cdot\}$ denotes the expectation. Evaluating the expectation with the expression for $y(k)$ in (7) leads to an expression for $r(\gamma)$, which does not depend on $k$, in terms of the AR coefficients and sin and cos terms related to the FO. Writing this expression out for several lag values $\gamma$ leads to the matrix equation

$$
\underline{r}=\left[\begin{array}{ll}
R & P \circ M
\end{array}\right] \underline{\psi}
$$

where $\underline{r}$ and $R$ are a vector and matrix containing values of $r(\gamma), P$ is a matrix containing sin and cos terms, $M$ is a matrix with terms related to the duration of the FO within the analysis window, and $\psi$ is a parameter vector containing the AR polynomial coefficients. The least squares solution to (11) is given by

$$
\underline{\hat{\psi}}=\left[\begin{array}{ll}
R & P \circ M
\end{array}\right]^{\dagger} \underline{r} .
$$

Implementing this solution requires estimates of the FO frequencies and durations to create $P$ and $M$.
Estimators for this purpose are proposed in [9, 10]. Once $\hat{\psi}$ is found, estimates of the AR polynomial coefficients can be inserted into (3)-(6) to estimate the frequency and damping ratio of modes.

The authors of [7] demonstrate that (11) holds approximately when the $M$ matrix is omitted. The performance of the algorithm with this approximation is evaluated using field-measured data in Section 4.

\section{Considerations for Real-World Implementation}

The authors of $[6,7]$ relied primarily on simulation results to demonstrate the reliability of $\mathrm{LS}+\mathrm{S}$ and $\mathrm{YW}+\mathrm{S}$. Given the promising results, the methods have since been applied to several months of field-measured PMU data. In the process, modifications were made to supporting algorithms that detect FOs and estimate their parameters to improve performance under real-world conditions. These modifications are described in the following subsections.

\subsection{Forced Oscillation Detection}

For $\mathrm{LS}+\mathrm{S}$ and $\mathrm{YW}+\mathrm{S}$ to account for FOs, the oscillations must first be detected. The results in this paper were generated by applying the FO detection algorithm proposed in [10]. The detector operates by identifying frequencies where the input signal's energy is higher than the underlying ambient noise energy. The detector operates in the frequency domain and depends on an estimate of the ambient noise spectrum. This estimate was obtained using the algorithm in [11], which is able to estimate the ambient spectrum despite the presence of FOs.

As described in [10], analyzing multiple segments of data with various lengths can improve detection performance. Shorter detection segments detect oscillations with large amplitudes quickly, while longer segments reliably detect small but persistent FOs. The results in Section 4 were obtained using the segment lengths depicted in Fig. 2, which were designed using the algorithm proposed in [10]. The use of multiple detection segments can lead to the same FO being detected several times, often with slightly different frequencies. These results can be combined to reflect a single FO, as described in [10].

One challenge with using the ambient noise estimator in [11] for multiple detection segments is that the estimator is only suitable for relatively long record lengths, say more than 10 minutes. Note that three of the detection segments in Fig. 2 fall below this limit. Thus, the ambient noise spectrum was first estimated using the most recent 30 minutes of data. Linear interpolation was 


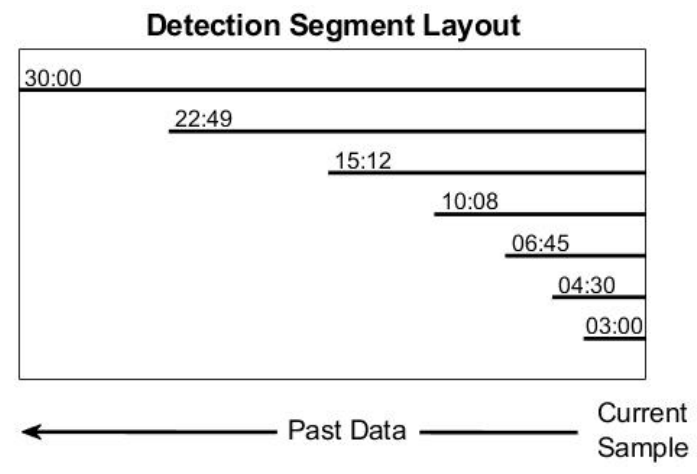

Figure 2. Detection segment lengths used for all reported results.

then used to obtain spectral estimates with appropriate frequency bins for each detection segment. Prior to the study, it was unknown if this approach would lead to undesirable detector performance. Results indicate that the approach is suitable for practical use with field-measured data.

The frequency range to examine for FOs must also be considered. The detector's sensitivity increases as the frequency range is narrowed [10], but the frequency range must be wide enough to capture all FOs that could bias the mode meter. The results in Section 4 focus on the WNAPS's North-South B mode, which typically varies between 0.32 and $0.42 \mathrm{~Hz}$ [12]. To ensure that FOs close enough to cause bias were detected, the frequency range from 0.25 to $0.5 \mathrm{~Hz}$ was examined.

\subsection{Forced Oscillation Frequency Estimation}

Forced oscillations are often detected by more than one of the detection segments in Fig. 2. These detection results can be combined to assign each FO a single frequency estimate [10]. This approach worked well with $\mathrm{YW}+\mathrm{S}$, but initial tests of $\mathrm{LS}+\mathrm{S}$ with field-measured data revealed problems with sensitivity to frequency errors. As will be demonstrated in Section 4, the performance of $\mathrm{LS}+\mathrm{S}$ was improved by not combining FOs detected by multiple detection segments. This approach relies on one of many frequency estimates being close to the true oscillation frequency, rather than relying on a single best estimate. The approach is marginally effective, but it has downsides. In particular, the processing time to perform time localization and mode estimation are increased.

The author of [13] demonstrates that the performance of the $\mathrm{LS}+\mathrm{S}$ algorithm can also be improved by refining the detector's FO frequency estimates. The results in this paper were generated using the iterative frequency estimation technique

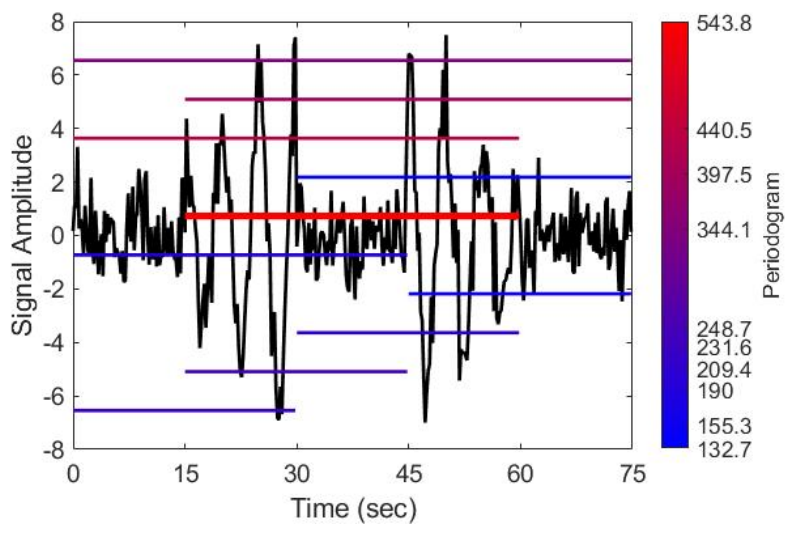

Figure 3. Visualization of time-localization results for an oscillation with varying amplitude and multiple occurrences.

proposed in [14], which was also used in [13].

\subsection{Forced Oscillation Time Localization}

Recall from Section 2 that $\mathrm{LS}+\mathrm{S}$ and $\mathrm{YW}+\mathrm{S}$ incorporate the FO's start and end points. To support these algorithms, a time localization algorithm was proposed in [9]. The method is limited in practical applications because it cannot account for FOs that are intermittent or have variable amplitudes.

For the results presented in this paper, an alternative method based on the periodogram was implemented. The periodogram is a measure of a signal's power as a function of frequency. In the time localization approach, several periodograms are calculated at the frequency of the FO for various windows of input measurements. The FO is then localized to the window resulting in the largest periodogram value.

For the results presented in this paper, a minimum window length of 60 seconds was selected. The window length was incremented in steps of 30 seconds, up to the 30 minute window used for the mode meter. For each evaluated length, the window was moved forward in 30 -second steps. In this way, a wide variety of window lengths and positions were evaluated. Results from a simplified example with two occurrences of a FO with varying amplitudes are presented in Fig. 3. In the figure, horizontal lines span the ranges of windows used to calculate the periodogram. Line colors correspond to the value of the periodogram at the oscillation's frequency. The bright red line most closely matches the location of the oscillations and therefore takes on the largest value. The two separate occurrences cannot be distinguished, but the algorithm does identify the window with the strongest evidence of a FO. 


\subsection{Forced Oscillation Tracking}

The processes described in Sections 3.1, 3.2, and 3.3 for detecting a FO and identifying its frequency, start time, and end time must be performed each time the mode estimate is to be updated. The results in Section 4 were generated by updating the mode estimate every five seconds. During initial testing, only FO detection and identification results from the current analysis window were incorporated. This approach is relatively straightforward to implement, but it has a significant drawback in that the mode estimates can vary significantly from one update to the next if a FO cannot be detected reliably.

To avoid this problem, the implementation was modified to take advantage of FO detection and identification results from the recent past. Once a FO is detected, it's start and end point are tracked until it leaves the mode meter's analysis window. The primary drawback of tracking FOs is that falsely detected oscillations can remain part of the ARMA+S model for significantly longer. Simulation results have shown that $\mathrm{LS}+\mathrm{S}$ is relatively insensitive to falsely detected FOs [6], and similar observations have been made for $\mathrm{YW}+\mathrm{S}$. Still, mode estimation performance can degrade when excessive false alarms occur, so it is important to tune the FO detector appropriately.

\section{Results from Field-Measured Data}

The modified mode meter algorithms described in Section 2, along with the supporting FO analysis algorithms in Section 3, were implemented in the open-source Archive Walker tool for testing. The Archive Walker tool is described in [15]. Additional documentation and software downloads, including the underlying MATLAB code, are available in [16].

The PMU data considered in this study was collected from the WNAPS in 2019 and spanned 10 months. An input signal approximating the difference in frequency between two areas of the system was designed to provide good observability of the North-South B mode. The detector from [10] was first applied to identify FOs with frequencies close to the mode. In particular, FOs that persisted for over an hour were sought for their potential to bias mode meters for significant lengths of time. The unmodified LS and YW algorithms were applied for these time periods to identify cases where bias occurred. Four such cases were selected for detailed analysis.

The settings for the mode meters and supporting FO detection, frequency estimation, time localization, and tracking algorithms were adjusted to achieve consistent performance across the four cases. Settings were also adjusted based on two six-hour sets of ambient data (no FOs present). The FO detector's performance was balanced to detect FOs as quickly as possible without producing excessive false alarms.

The FO amplitude estimates reported for each case are based on three algorithms. The $\mathrm{LS}+\mathrm{S}$ and $\mathrm{YW}+\mathrm{S}$ algorithms return $\mathrm{FO}$ amplitude estimates as part of their parameter vectors (see (9) and (12)) [6, 17]. The third amplitude estimator is based on how much the input signal's energy exceeds the ambient noise energy in the FO detector. Details can be found in [17]. Based on estimates from the three algorithms, an amplitude range and associated signal-to-noise ratio (SNR) are reported for each FO case. For the SNR, the FO is considered the signal embedded in the PMU's frequency measurement.

\subsection{Case 1}

The first case spanned 1.5 hours. During this time, a FO was detected within the mode meter's analysis window for approximately 40 minutes. The oscillation's amplitude was estimated between 0.076 and $0.130 \mathrm{mHz}$, corresponding to an SNR between -14 and $-9 \mathrm{~dB}$. Frequency estimates for the FO that caused bias are displayed in the top plot of Fig. 4. The figure's middle plot indicates the length of the associated detection segment. Note that all seven of the detection segments contribute to tracking the FO, with shorter detection segments offering early detection and longer detection segments providing more consistent frequency estimates. The bottom plot displays the time localization results. For each time along the $\mathrm{x}$ axis, the black portion indicates the FO's estimated position within the mode meter's analysis window. The FO is first detected in the mode meter window's newest samples. As time progresses, the analysis window slides over the FO until it leaves the analysis window entirely just before hour 1 . The mode meter algorithms were applied based on these estimates of frequency, start time, and end time.

The resulting mode estimates from the YW and LS algorithms are displayed in Fig. 5 and 6, respectively. In Fig. 5, results from the unmodified YW algorithm are shown in green. The damping ratio estimates are biased low for approximately 40 minutes while the FO is in the mode meter's analysis window. Results from $\mathrm{YW}+\mathrm{S}$ using the time-localization estimates in Fig. 4 are shown in red, while results using the approximation that does not depend on the FO's duration are shown in blue. For both implementations, the bias is significantly reduced. The two implementations generally show good agreement, though the time localization estimates introduce sudden jumps in the 

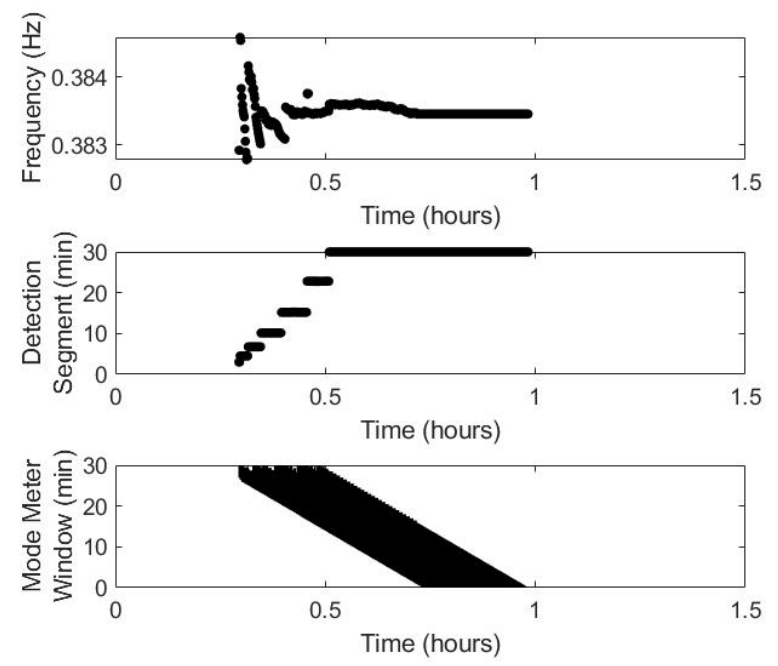

Figure 4. Detection results for Case 1 including estimated frequencies (top), associated detection segment (middle), and time localization (bottom).

mode estimates, particularly frequency. The sudden increase in the damping ratio estimates when the FO is first detected indicates that there was a several minute delay before the FO was detected.

Like its YW counterpart, the unmodified LS algorithm produced biased mode estimates while the FO was present, as indicated by the green lines in Fig. 6. The LS+S algorithm was able to largely avoid the bias in this case. Mode estimates in blue were obtained using frequency estimates from all detection segments, while those in red were obtained by combining detection results into a single best estimate of the oscillation's frequency.

The bias introduced into the mode estimates is significant, especially considering the FO's small amplitude. Though the detection algorithm did not detect it immediately, it was fast enough to prevent severe bias from the modified mode meter algorithms. To demonstrate the difficulty of detecting such small oscillations, consider the plot of the input signal's RMS-energy in Fig. 7. This RMS-energy was calculated for the frequency band between 0.1 and $1 \mathrm{~Hz}$ using the method described in [18]. Note that any increase in RMS-energy due to the FO would be impossible to distinguish from the surrounding ambient noise. Similar results were obtained for the other three cases considered in this paper. The RMS-energy method has been used with great success by industry to monitor for FOs threatening reliability $[5,4]$. The objective here is to demonstrate that alternative detection approaches are necessary for this application.
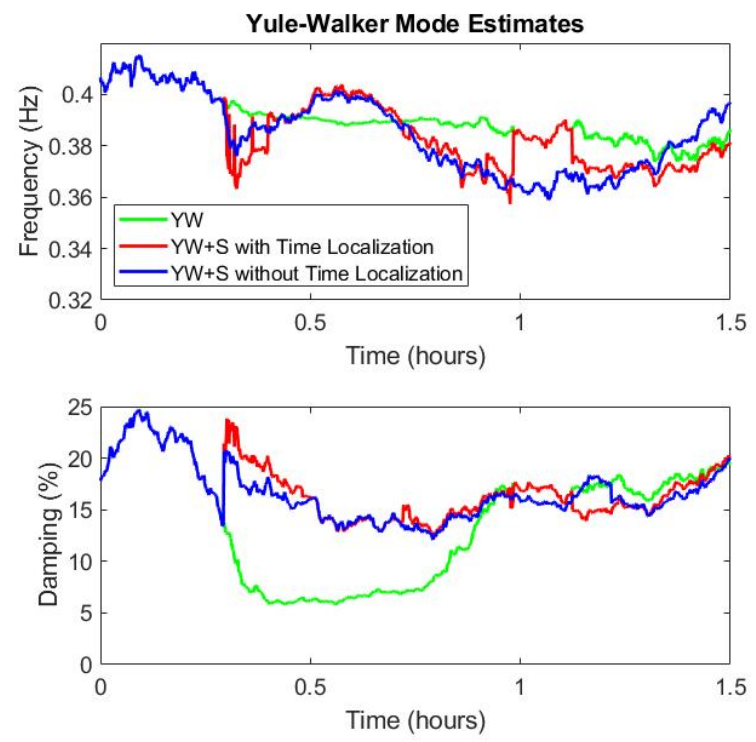

Figure 5. Mode estimation results from the Yule-Walker algorithms for Case 1.
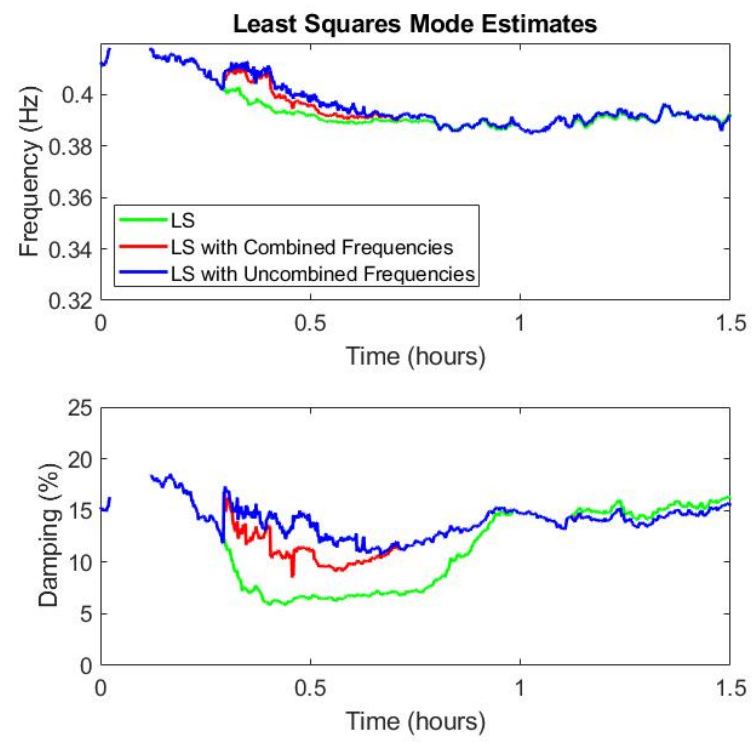

Figure 6. Mode estimation results from the least squares algorithms for Case 1.

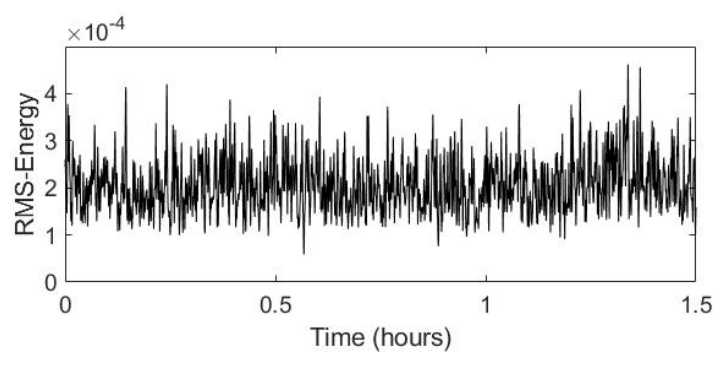

Figure 7. RMS-energy of the input signal for Case 1. 

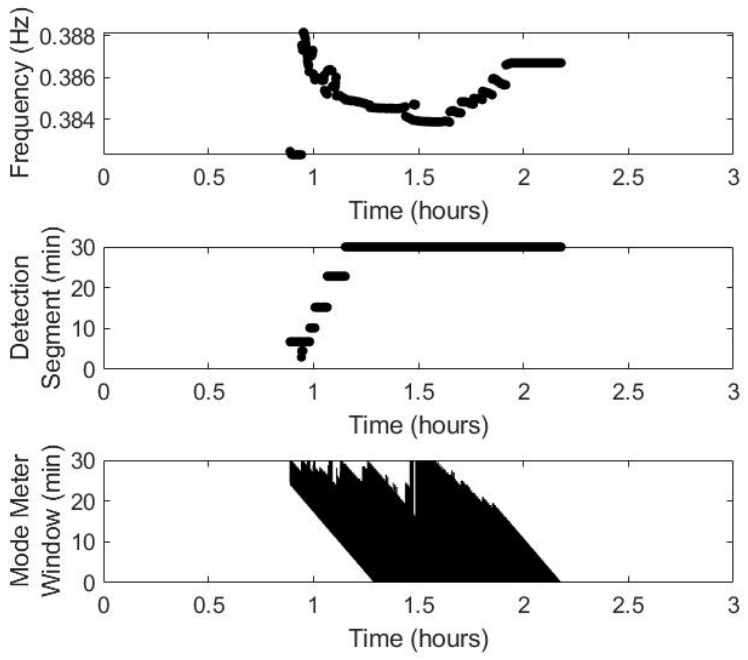

Figure 8. Detection results for Case 2 including estimated frequencies (top), associated detection segment (middle), and time localization (bottom).

\subsection{Case 2}

The second case spanned three hours with a FO present in the mode meter analysis window for approximately 1.25 hours. The oscillation's amplitude was estimated between 0.060 and $0.155 \mathrm{mHz}$, which corresponded to an SNR between -16 and $-7 \mathrm{~dB}$. A gradual variation was observed in the amplitude and frequency estimates. This variation can be clearly seen in the top plot of Fig. 8. The bottom plot shows that time localization estimates were less consistent than for Case 1.

Mode meter estimates for the YW algorithms are plotted in Fig. 9. Note that the frequency estimates from the unmodified algorithm track the FO's varying frequency, which is a strong indicator of bias. The damping ratio estimates are also clearly suppressed. The estimates from $\mathrm{YW}+\mathrm{S}$ are already suppressed when the FO is detected, but the worst bias is avoided. The frequency estimates jump initially, probably because of the poor frequency estimates apparent early in Fig. 8.

In the case of the LS algorithms, only the implementation using frequency estimates from all detection segments avoided extensive bias. Due to the sensitivity of $\mathrm{LS}+\mathrm{S}$ to errors in the FO frequency, severe bias resulted when a single combined frequency was used because one value could not represent the FO's varying frequency.
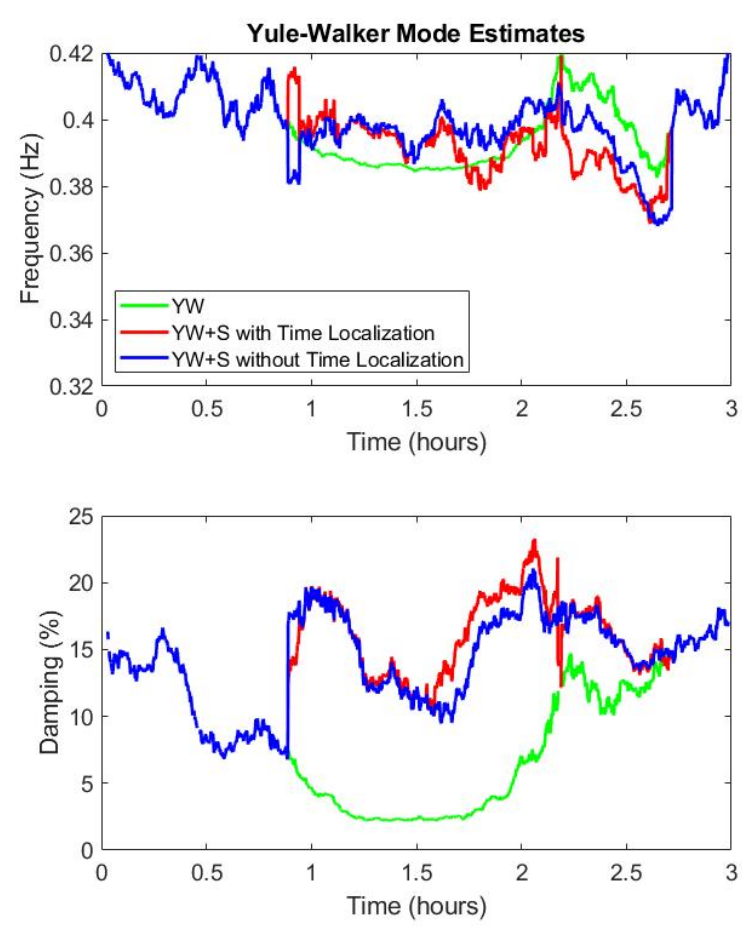

Figure 9. Mode estimation results from the Yule-Walker algorithms for Case 2.
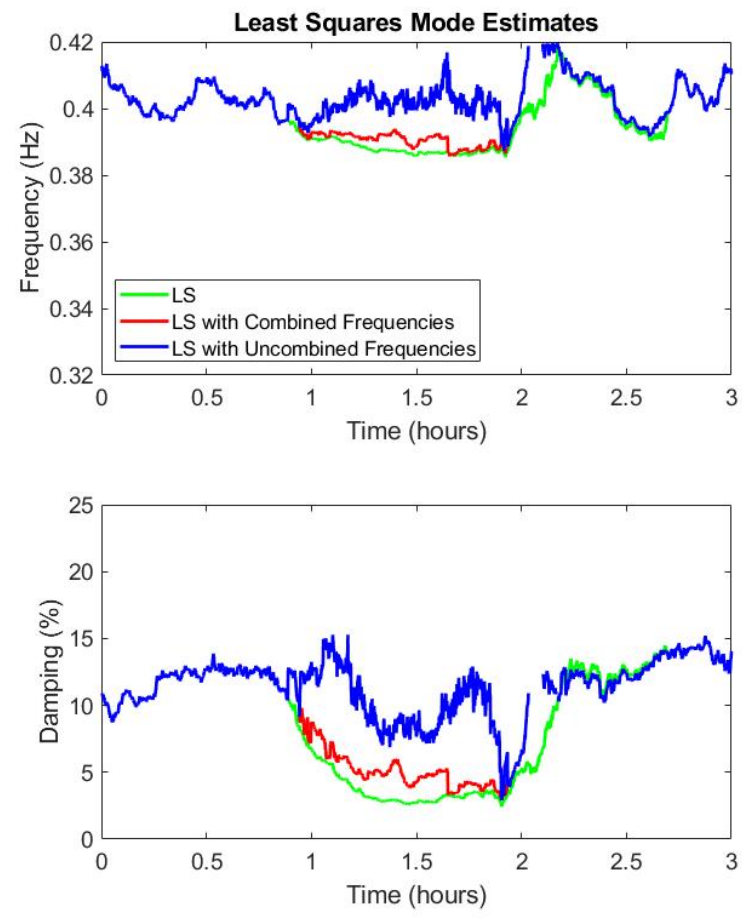

Figure 10. Mode estimation results from the least squares algorithms for Case 2. 

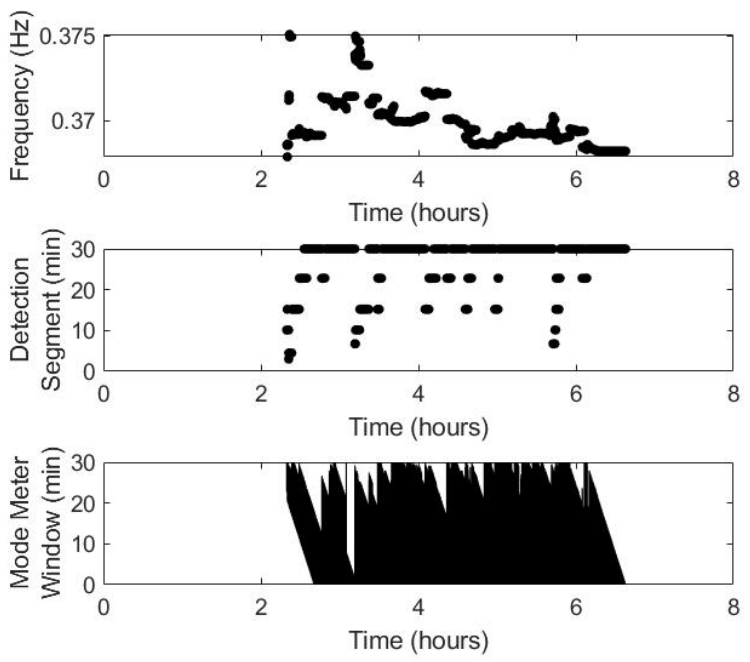

Figure 11. Detection results for Case 3 including estimated frequencies (top), associated detection segment (middle), and time localization (bottom).

\subsection{Case 3}

The third case was the longest, spanning eight hours with a FO that biased mode meter estimates for over four hours. The oscillation's amplitude was estimated between 0.068 and $0.098 \mathrm{mHz}$, which corresponded to an SNR between -14 and $-11 \mathrm{~dB}$. Detection results are plotted in Fig. 11. In Cases 1 and 2, the length of the segment detecting the oscillation steadily increased. This pattern repeats several times in the middle plot of Fig. 11, indicating that the FO likely stopped and started several times during the analysis. As a consequence, the time localization results in the bottom plot of Fig. 11 are more difficult to interpret than in previous cases and probably less accurate.

Despite the complexities of this case, Fig. 12 shows that $\mathrm{YW}+\mathrm{S}$ avoids most of the bias present in the results from the unmodified version. Once again, including the time localization estimates makes little difference in the mode estimates. Mode estimates are missing between hours 4 and 5 because the estimates go above the $25 \%$ damping ratio threshold for tracking.

As depicted in Fig. 13, LS+S does not perform well for this case. The bias in the damping ratio estimates is reduced for certain periods, but there are long periods where the bias for $\mathrm{LS}+\mathrm{S}$ is almost as severe as for the unmodified LS. This poor performance is likely due to the sensitivity of $\mathrm{LS}+\mathrm{S}$ to errors in time localization and FO frequency estimates.
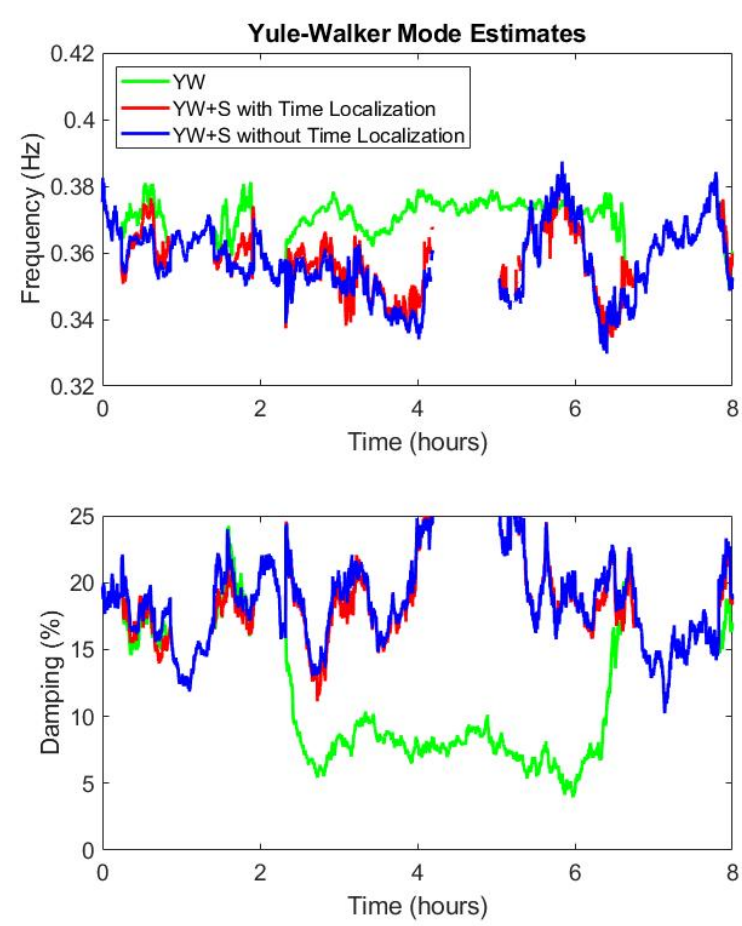

Figure 12. Mode estimation results from the Yule-Walker algorithms for Case 3.
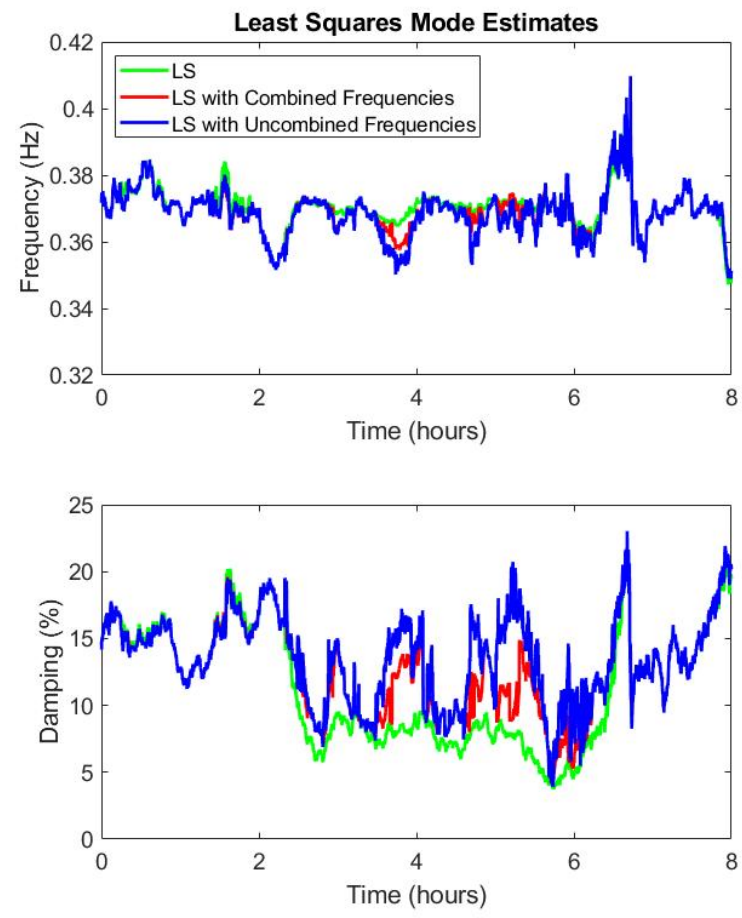

Figure 13. Mode estimation results from the least squares algorithms for Case 3 . 

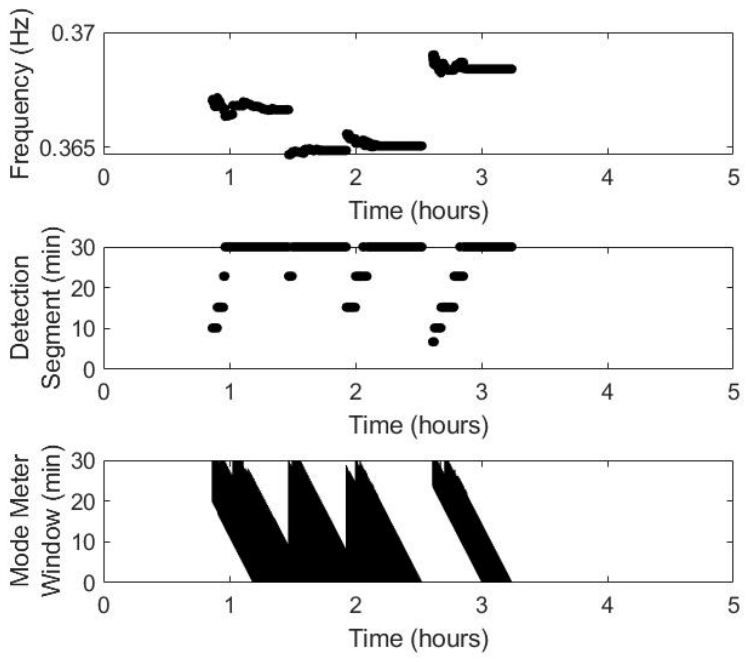

Figure 14. Detection results for Case 4 including estimated frequencies (top), associated detection segment (middle), and time localization (bottom).

\subsection{Case 4}

The fourth case spanned five hours and contained a FO that biased mode estimates for approximately three hours. The FO's amplitude was estimated between 0.072 and $0.094 \mathrm{mHz}$, corresponding to an SNR between -14 and $-11 \mathrm{~dB}$. The detection results in Fig. 14 show that as with Case 3, the FO appears to have multiple occurrences. In this case, the FO frequencies differ between occurrences.

As shown in Fig. 15, the mode estimates become biased for over 30 minutes before the FO is detected. Once detected, the mode estimate bias from $\mathrm{YW}+\mathrm{S}$ is reduced significantly. Note that at approximately hour 2.5 the modified algorithm using time localization estimates becomes severely biased for approximately 10 minutes. This result points to the advantages of removing dependence on the time localization results.

Estimates from the LS algorithms are shown in Fig. 16. As with Case 3, the poor performance is likely due to time localization and FO frequency estimate errors.

\subsection{Discussion}

Results from the four cases demonstrate the real-world potential for FOs to bias existing mode meters. Bias can occur even for low-amplitude FOs, which cannot be identified by methods currently in use to detect FOs threatening system reliability, as shown in Fig. 7. Thus, alternative mode meter algorithms and
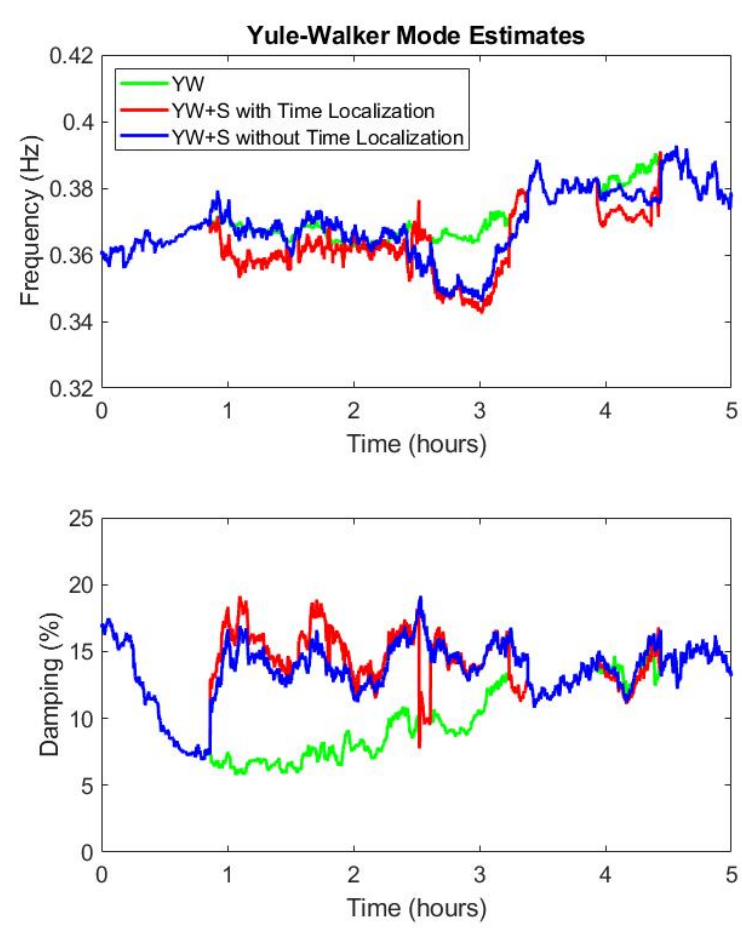

Figure 15. Mode estimation results from the Yule-Walker algorithms for Case 4.
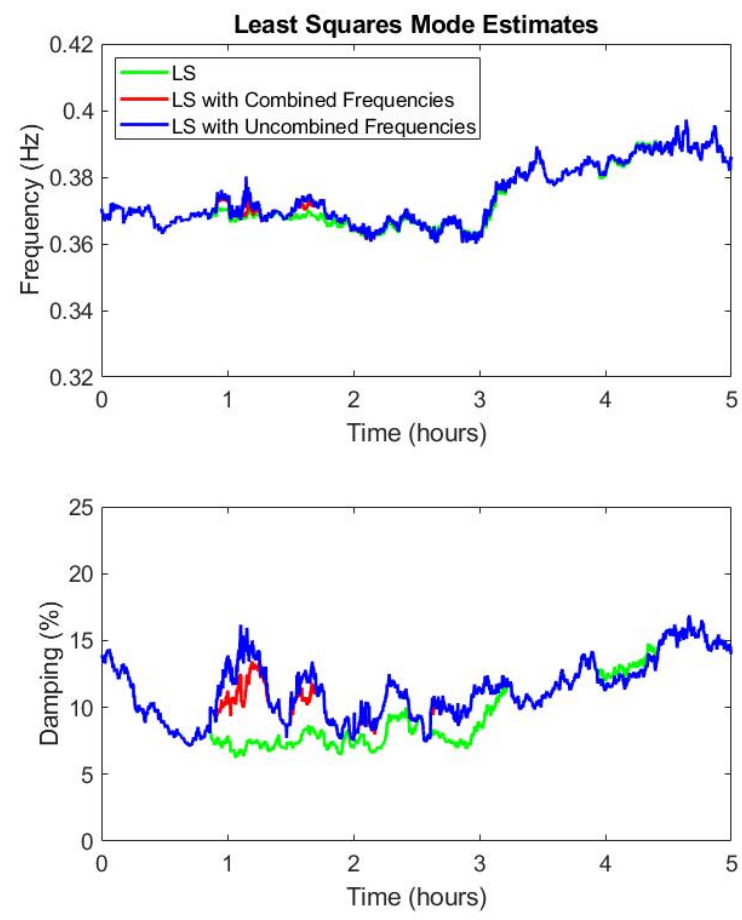

Figure 16. Mode estimation results from the least squares algorithms for Case 4. 
supporting FO detectors are needed.

The forced oscillation detector proposed in [10] was shown to provide sufficient support to the $\mathrm{YW}+\mathrm{S}$ algorithm. Detection results also showed that real-world FOs exhibit complex behavior, such as varying frequencies and amplitudes. Though the time localization algorithm proposed here is more robust than the method in [9], it is still insufficient to handle these complexities. The $\mathrm{LS}+\mathrm{S}$ algorithm does not appear well-suited to use in real-world conditions due to its dependence on the time localization estimates and its severe sensitivity to errors in FO frequency estimates.

The YW+S algorithm, however, does appear robust enough to provide reliable mode estimates in real-world conditions. Results indicate that the approximation in [7] that removes the algorithm's dependence on FO duration estimates holds for field-measured data. The ability to avoid time localization is a significant advantage because it is computationally intensive and difficult in real-world conditions. Thus, the $\mathrm{YW}+\mathrm{S}$ algorithm implemented without FO duration estimates is the most promising algorithm for practical deployment.

\section{Conclusion}

Power system reliability depends on maintaining the system's small-signal stability. Through continuous monitoring of electromechanical modes of oscillation, mode meters help system operators maintain stability. However, conventional mode meter algorithms can be biased by forced oscillations, leading to unreliable performance. In this paper, four such cases were examined. The performance of modified least squares and Yule-Walker mode meter algorithms, along with the forced oscillation detection and identification algorithms on which they depend, were evaluated using these cases. Results indicate that the LS+S and time localization algorithms are unreliable for real-world conditions. The $\mathrm{YW}+\mathrm{S}$ algorithm performed well and does not require time localization results. It therefore shows promise for practical deployment.

\section{References}

[1] D. N. Kosterev, C. W. Taylor, and W. A. Mittelstadt, "Model validation for the August 10, 1996 WSCC system outage," Power Systems, IEEE Transactions on, vol. 14, no. 3, pp. 967-979, 1999.

[2] J. W. Pierre, D. J. Trudnowski, and M. K. Donnelly, "Initial results in electromechanical mode identification from ambient data," Power Systems, IEEE Transactions on, vol. 12, no. 3, pp. 1245-1251, 1997.

[3] A. Agarwal, J. Balance, B. Bhargava, J. Dyer, K. Martin, and J. Mo, "Real time dynamics monitoring system (RTDMS®) for use with synchrophasor technology in power systems," in 2011 IEEE Power and Energy Society General Meeting, pp. 1-8, 2011.

[4] D. Kosterev, J. Burns, N. Leitschuh, J. Anasis, A. Donahoo, D. Trudnowski, M. Donnelly, and J. Pierre, "Implementation and operating experiences with oscillation detection application at Bonneville Power Administration," in Proceedings of CIGRE 2016 Grid of the Future Conference, pp. 1-12, 2016.

[5] H. Zhang, J. Ning, H. Yuan, and V. Venkatasubramanian, "Implementing online oscillation monitoring and forced oscillation source locating at Peak Reliability," in 2019 North American Power Symposium (NAPS), pp. 1-6, 2019.

[6] J. Follum, J. W. Pierre, and R. Martin, "Simultaneous estimation of electromechanical modes and forced oscillations," IEEE Transactions on Power Systems, vol. 32, no. 5, pp. 3958-3967, 2017.

[7] U. Agrawal, J. Follum, J. W. Pierre, and D. Duan, "Electromechanical mode estimation in the presence of periodic forced oscillations," IEEE Transactions on Power Systems, vol. 34, no. 2, pp. 1579-1588, 2019.

[8] M. Maddipour Farrokhifard, M. Hatami, and V. M. Venkatasubramanian, "Performance of stochastic subspace identification methods in presence of forced oscillations," in 2019 International Conference on Smart Grid Synchronized Measurements and Analytics (SGSMA), pp. 1-8, 2019.

[9] J. Follum and J. Pierre, "Time-localization of forced oscillations in power systems," in 2015 IEEE Power Energy Society General Meeting, pp. 1-5, 2015.

[10] J. Follum and J. W. Pierre, "Detection of periodic forced oscillations in power systems," IEEE Transactions on Power Systems, vol. 31, no. 3, pp. 2423-2433, 2016.

[11] J. Follum, F. Tuffner, and U. Agrawal, "Applications of a new nonparametric estimator of ambient power system spectra for measurements containing forced oscillations," in 2017 IEEE Power Energy Society General Meeting, pp. 1-5, 2017.

[12] "Modes of inter-area power oscillations in western interconnection," tech. rep., WECC Joint Synchronized Information Subcommittee, 2013.

[13] L. Dosiek, "The effects of forced oscillation frequency estimation error on the LS-ARMA+S mode meter," IEEE Transactions on Power Systems, vol. 35, no. 2, pp. 1650-1652, 2020.

[14] E. Aboutanios and B. Mulgrew, "Iterative frequency estimation by interpolation on Fourier coefficients," IEEE Transactions on Signal Processing, vol. 53, no. 4, pp. 1237-1242, 2005.

[15] J. Follum, P. Etingov, F. Tuffner, H. Wang, U. Agrawal, D. Kosterev, S. Yang, and A. Faris, "Detecting and analyzing power system disturbances in PMU data with the open-source Archive Walker tool," in IEEE/PES Transmission and Distribution Conference and Exhibition, 2020, accepted.

[16] PNNL, "Setting up and reviewing analyses with the Archive Walker GUI." [Online]. Available: https://github.com/pnnl/archive_walker/wiki, 2018.

[17] J. Follum, Electromechanical Mode Estimation in the Presence of Forced Oscillations. PhD thesis, University of Wyoming, 2014.

[18] M. Donnelly, D. Trudnowski, J. Colwell, J. Pierre, and L. Dosiek, "RMS-energy filter design for real-time oscillation detection," in 2015 IEEE Power Energy Society General Meeting, pp. 1-5, 2015. 